\title{
Evaluation of the Beauveria bassiana Grown under Nanomaterial Enriched Media for its Relative Efficacy against $S$. litura under Laboratory Conditions
}

\author{
M. Gayathri ${ }^{1 *}$, N.C. Venkateswarlu ${ }^{1}$, T. Murali Krishna ${ }^{2}$ and K. Devaki ${ }^{1}$ \\ ${ }^{1}$ Department of Entomology, S.V. Agricultural College, Tirupati 517502, India \\ ${ }^{2}$ KVK, Kalyandurg, Ananthapur district, 515761, India \\ *Corresponding author
}

\section{A B S T R A C T}

Key w or d s
$\begin{aligned} & \text { Nanomaterials, } \\ & \text { Beauveria bassiana, } \\ & \text { S. litura relative } \\ & \text { efficacy }\end{aligned}$
Article Info
$\begin{aligned} & \text { Accepted: } \\ & \text { 15 November } 2018 \\ & \text { Available Online: } \\ & \text { 10 December } 2018\end{aligned}$

\section{Introduction}

The tobacco caterpillar, Spodoptera litura (F.), has been reported as one of the major insect pest of groundnut and feed on 112 cultivated food plants all over the world (Mousa et al., 1980) of which 40 are grown in India (Basu, 1981, Muthukrishnan et al., 2005). It passes through 5-6 overlapping generations annually (Sasidharan and Varma, 2005; Kumar and Chapman, 2006) and if not controlled timely, it may causes in huge crop losses ranging from 25.8-100 percent in various parts of India (Ahmad et al., 2005).
The management of $S$. litura using insecticides has become difficult because of the development of resistance and effect to non-target organisms viz., natural enemy population as well as frequent use of these insecticides increasing problems of human health and environmental pollution.

Biological control of insect pests is one of the most important component of Integrated Pest Management (IPM), wherein entomopathogens such as bacteria, viruses and fungi are exploited against insect pests. 
Although 700 to 750 species of EPF have been reported as pathogenic to insects but only about a dozen have been exploited for insect control (Stark and Banks, 2003). Among these Beauveria bassiana (Balsamo) Vuillemin (Ascomycota: Hyphocreales) is a facultative pathogen with wide host range (Armes et al., 1997; Sahayaraj et al., 2007). This fungus has potential to control over 70 insect pests belonging to different orders particularly lepidopteran pests, infesting various crops and appears to be innocuous to most non target organisms.

The bioefficacy of entomopathogens in relation to number of conidia in B.bassiana were chance to increase the bioefficacy with respect to the mortality of lepidopteran larvae by adding minerals. These minerals are namely calcium, magnesium, iron and zinc will enhance the conidial count in B.bassiana. As a result the efficacy of entomopathogens will increases. The nanoparticles of these minerals will increase the conidial count in B.bassiana and also chance to decrease the dose of biopesticides. Nanoparticles are atomic or molecular aggregates characterized by size less than $100 \mathrm{~nm}$. These are actually modified form of basic elements derived by altering their atomic as well as molecular properties of elements (Suchea, et al., 2006).

To enhance the biopesticides efficacy interms of increasing the number of conidial in B.bassiana the mineral salts viz., Calcium, magnesium, iron and zinc were added to the media before inoculation (Valicente et al., 2010).

By considering all these issues, to know the impact of nanobased material on bio pesticides to enhance their efficacy for longer periods and their combined effect on S.litura management, the present study has been taken up.

\section{Materials and Methods}

\section{Preparation of nanoparticulate solutions}

Oxide nanoparticles of $\mathrm{Zn}, \mathrm{Ca}, \mathrm{Mg}$ and $\mathrm{Fe}$ weighing $250 \mathrm{mg}$ was added to $500 \mathrm{ml}$ of distilled water $(500 \mathrm{ppm})$ and from this solution different concentrations (100, 50, 20 and $10 \mathrm{ppm}$ ) of nanoparticulate solutions were prepared by adding the respective volumes of distilled water.

From the prepared nanoparticulate solutions $\mathrm{Zn}, \mathrm{Fe}, \mathrm{Ca}$ and $\mathrm{Mg}$ at $10 \mathrm{ppm}, 20 \mathrm{ppm}, 50$ ppm, $100 \mathrm{ppm}$ and $500 \mathrm{ppm}$ in $1: 9$ ratio $(1 \mathrm{ml}$ of nanoparticulate solution to $9 \mathrm{ml}$ of LBA media) was added to the Sabouraud Dextrose Agar media (SDAY) media before sterilisation to study the relative efficacy of B.bassiana against S.litura.

\section{Mass multiplication of S. litura}

For multiplication and maintenance of S.litura population in the laboratory equipment like wire cages for oviposition, plastic troughs for larval maintenance, plastic boxes for pupae were cleaned with ethanol and were dried under solar radiation. The mother culture of S.litura egg masses were collected from field and surface sterilized with 4 per cent formaldehyde, washed 3-4 times with distilled water and kept in plastic transparent covers tied with rubber band. Castor leaves were provided to freshly emerged Spodoptera larvae. After three days, the larvae were segregated based on size into transparent plastic rearing troughs and covered with muslin cloth. The food material was changed twice in a day till pupation. Before pupation, larvae were transferred to another plastic container containing sterilized soil and allowed them for pupation. The emerged moths were transferred to oviposition cages. Cotton swab dipped in 10 per cent honey solution was kept in cage as food for the 
adults. For oviposition by the female moths fresh castor plant with tender twigs were arranged in conical flasks containing $3 / 4^{\text {th }}$ of water and kept in cages. Egg masses were collected every day and later sterilized. After hatching, the larvae were reared up to third instar for further laboratory bioassay studies.

\section{Bioassay of B.bassiana grownon Nanomaterial based media against $S$. litura}

To know the efficiency of B.bassiana grown on nanomaterial based media, bioassay studies were conducted in laboratory against S.litura. The spore suspension was prepared from 15 days old culture of B.bassiana along with added nanomaterials viz., $\mathrm{Zn}, \mathrm{Ca}, \mathrm{Mg}$ and $\mathrm{Fe}$ at $10,20,50,100$ and $500 \mathrm{ppm}$ concentration and without nanomaterials was evaluated against S.litura on $3^{\text {rd }}$ instar larvae at 10 days interval. Sterilized plastic troughs $(20 \mathrm{~cm} \times 20$ $\mathrm{cm})$ were taken into which one groundnut compound leaf containing four leaflets (quadrate leaf) was dipped for 10 minutes into culture broth $\left(1 \times 10^{8}\right.$ to $1 \times 10^{5}$ spores $\left.1 \mathrm{~mL}^{-1}\right)$, later leaflets were taken out and kept for air drying till leaf surface free from moisture. After drying, the petiole of leaf was swabbed with wet cotton to maintain leaf succulence and turgidity. One compound leaf was used for one replication. which was placed in a Petri plate. Ten larvae were released per each treatment which was replicated thrice. In control treatment the leaflets (quadrat leaf) were dipped in distilled water and served as control. The larval mortality was assessed after $120 \mathrm{~h}$ at regular intervals.

For each treatment ten third instar S.litura larvae were released to crawl on the treated leaf and daily observations were recorded on post treatment changes of larvae, larval mortality, pupal mortality, malformed pupae and adults until to death. Each treatment was replicated thrice along with an untreated control (Hafez et al., 1994). The per cent larval mortality was expressed by using the following formula.

The larval mortality was converted to percentage before subjecting to statistical analysis by using the formula.

Per cent larval mortality $=$

No. of larvae dead due to infection

Total number of larvae treated

The per cent mortality was analysed statistically after transforming into angular values.

\section{Results and Discussion}

Evaluation of $B$. bassiana grown in nanomaterial enriched media against $S$. litura

B. bassiana (SGB strain) culture was maintained in the laboratory and $5 \mathrm{~mm}$ discs were made from four days old culture and inoculated in to SDAY plates which were added with different nanomaterials at different concentrations. Then the culture was allowed to grow for 15 days. Then spore suspension was prepared by mixing with $10 \mathrm{ml}$ of sterile distilled water. A series of bioassays were conducted by providing groundnut leaves which were dipped into spore suspensions containing B.bassiana. Then the mortality rate was recorded daily up to pupation and results are presented in the table 1 and 2 .

During 2016, the mortality of S.itura after 120 hours of treatment was ranged from 6.67 to 76.67 per cent. The maximum mortality of 76.67 per cent was recorded with $\mathrm{MgO}$ based B.bassiana at $50 \mathrm{ppm}$ and 73.33 with $\mathrm{CaO}$ based B.bassiana, at $20 \mathrm{ppm}$ and 56.67 with $\mathrm{ZnO}$ based B.bassiana and 20.00 and 43.33 per cent with $\mathrm{FeO}$ based B.bassiana. 
Table.1 Influence of different nanoparticles on the efficacy of B.bassiana against S.litura at different concentrations during the year 2016

\begin{tabular}{|c|c|c|c|c|c|c|}
\hline \multirow[t]{2}{*}{ S.No. } & \multirow[t]{2}{*}{ Name of the Treatment } & \multicolumn{5}{|c|}{ S.litura Per cent mortality } \\
\hline & & $120 h$ & $144 h$ & $168 h$ & $192 \mathrm{~h}$ & Cumulative \\
\hline 1 & Magnesium oxide $(\mathrm{MgO}) 10$ ppm & $\begin{array}{c}36.67 \\
(33.21) \mathrm{cdef}\end{array}$ & $\begin{array}{c}23.33 \\
(28.88) \text { bcde }\end{array}$ & $\begin{array}{c}6.67 \\
(14.96)\end{array}$ & $\begin{array}{c}0.00 \\
(0.00)\end{array}$ & $\begin{array}{c}66.67 \\
(54.74) \mathrm{ab}\end{array}$ \\
\hline 2 & Magnesium oxide (MgO) 20 ppm & $\begin{array}{c}33.33 \\
(43.09) \mathrm{def}\end{array}$ & $\begin{array}{c}40.00 \\
(3923) \mathrm{ab}\end{array}$ & $\begin{array}{c}10.00 \\
(18.43)\end{array}$ & $\begin{array}{c}0.00 \\
(0.00)\end{array}$ & $\begin{array}{c}83.33 \\
(65.91) a b\end{array}$ \\
\hline 3 & Magnesium oxide $(\mathrm{MgO}) 50$ ppm & $\begin{array}{c}76.67 \\
(58.91) \mathrm{a}\end{array}$ & $\begin{array}{c}13.33 \\
(21.42) \mathrm{de}\end{array}$ & $\begin{array}{c}0.00 \\
(0.00)\end{array}$ & $\begin{array}{c}0.00 \\
(0.00)\end{array}$ & $\begin{array}{c}90.00 \\
(71.57) \mathrm{a}\end{array}$ \\
\hline 4 & Magnesium oxide $(\mathrm{MgO}) 100 \mathrm{ppm}$ & $\begin{array}{c}43.33 \\
(41.17) \mathrm{cd}\end{array}$ & $\begin{array}{c}30.00 \\
(33.21) \mathrm{abcd}\end{array}$ & $\begin{array}{c}6.67 \\
(14.96)\end{array}$ & $\begin{array}{c}0.00 \\
(0.00)\end{array}$ & $\begin{array}{c}80.00 \\
(63.43) \mathrm{ab}\end{array}$ \\
\hline 5 & Magnesium oxide $(\mathrm{MgO}) 500 \mathrm{ppm}$ & $\begin{array}{c}33.33 \\
\text { (35.26)def }\end{array}$ & $\begin{array}{c}40.00 \\
(39.22) \mathrm{ab}\end{array}$ & $\begin{array}{c}3.33 \\
(10.52)\end{array}$ & $\begin{array}{c}0.00 \\
(0.00)\end{array}$ & $\begin{array}{c}76.67 \\
(61.12) a b\end{array}$ \\
\hline 6 & Calcium oxide $(\mathrm{CaO}) 10 \mathrm{ppm}$ & $\begin{array}{c}23.33 \\
(28.88) \text { efg }\end{array}$ & $\begin{array}{c}46.67 \\
(49.09) \mathrm{a}\end{array}$ & $\begin{array}{c}6.67 \\
(14.96)\end{array}$ & $\begin{array}{c}0.00 \\
(0.00)\end{array}$ & $\begin{array}{c}76.67 \\
(61.12) \mathrm{ab}\end{array}$ \\
\hline 7 & Calcium oxide $(\mathrm{CaO}) 20 \mathrm{ppm}$ & $\begin{array}{c}73.33 \\
(58.91) \mathrm{a}\end{array}$ & $\begin{array}{c}16.67 \\
(24.09) \text { cde }\end{array}$ & $\begin{array}{c}0.00 \\
(0.00)\end{array}$ & $\begin{array}{c}0.00 \\
(0.00)\end{array}$ & $\begin{array}{c}90.00 \\
(71.57) \mathrm{a}\end{array}$ \\
\hline 8 & Calcium oxide $(\mathrm{CaO}) 50$ ppm & $\begin{array}{c}23.33 \\
(28.88) \text { efg }\end{array}$ & $\begin{array}{c}36.67 \\
(37.27) \mathrm{ab}\end{array}$ & $\begin{array}{c}16.67 \\
(24.09)\end{array}$ & $\begin{array}{c}0.00 \\
(0.00)\end{array}$ & $\begin{array}{c}76.67 \\
(61.12) \mathrm{ab}\end{array}$ \\
\hline 9 & Calcium oxide $(\mathrm{CaO}) 100$ ppm & $\begin{array}{c}30.00 \\
\text { (33.21)def }\end{array}$ & $\begin{array}{c}33.33 \\
(35.26) \mathrm{abc}\end{array}$ & $\begin{array}{c}10.00 \\
(18.43)\end{array}$ & $\begin{array}{c}0.00 \\
(0.00)\end{array}$ & $\begin{array}{c}73.33 \\
(58.91) a b\end{array}$ \\
\hline 10 & Calcium oxide $(\mathrm{CaO}) 500$ ppm & $\begin{array}{c}26.67 \\
\text { (31.09)def }\end{array}$ & $\begin{array}{c}33.33 \\
(35.26) \mathrm{abc}\end{array}$ & $\begin{array}{c}10.00 \\
(18.43)\end{array}$ & $\begin{array}{c}0.00 \\
(0.00)\end{array}$ & $\begin{array}{c}70.00 \\
(56.79) \mathrm{ab}\end{array}$ \\
\hline 11 & Zinc oxide $(\mathrm{ZnO}) 10 \mathrm{ppm}$ & $\begin{array}{c}43.33 \\
(41.17) \mathrm{cd}\end{array}$ & $\begin{array}{c}33.33 \\
(35.26) \mathrm{abc}\end{array}$ & $\begin{array}{c}6.67 \\
(14.96)\end{array}$ & $\begin{array}{c}0.00 \\
(0.00)\end{array}$ & $\begin{array}{c}83.33 \\
(65.91) a b\end{array}$ \\
\hline 12 & Zinc oxide $(\mathrm{ZnO}) 20 \mathrm{ppm}$ & $\begin{array}{c}53.33 \\
(46.91) b c\end{array}$ & $\begin{array}{c}16.67 \\
(24.09) \text { cde }\end{array}$ & $\begin{array}{c}10.00 \\
(18.43)\end{array}$ & $\begin{array}{c}0.00 \\
(0.00)\end{array}$ & $\begin{array}{c}76.67 \\
(61.12) \mathrm{ab}\end{array}$ \\
\hline 13 & Zinc oxide $(\mathrm{ZnO}) 50 \mathrm{ppm}$ & $\begin{array}{c}26.67 \\
\text { (31.09)def }\end{array}$ & $\begin{array}{c}36.67 \\
(37.27) \mathrm{ab}\end{array}$ & $\begin{array}{c}6.67 \\
(14.96)\end{array}$ & $\begin{array}{c}0.00 \\
(0.00)\end{array}$ & $\begin{array}{c}70.00 \\
(56.79) \mathrm{ab}\end{array}$ \\
\hline 14 & Zinc oxide $(\mathrm{ZnO}) 100 \mathrm{ppm}$ & $\begin{array}{c}30.00 \\
\text { (33.21)def }\end{array}$ & $\begin{array}{c}33.33 \\
(35.26) \mathrm{abc}\end{array}$ & $\begin{array}{c}13.33 \\
(21.42)\end{array}$ & $\begin{array}{c}0.00 \\
(0.00)\end{array}$ & $\begin{array}{c}83.33 \\
(65.91) a b\end{array}$ \\
\hline 15 & Zinc oxide $(\mathrm{ZnO}) 500 \mathrm{ppm}$ & $\begin{array}{c}20.00 \\
(26.57) \mathrm{fg}\end{array}$ & $\begin{array}{c}33.33 \\
(35.26) \mathrm{abc}\end{array}$ & $\begin{array}{c}13.33 \\
(21.42)\end{array}$ & $\begin{array}{c}0.00 \\
(0.00)\end{array}$ & $\begin{array}{c}80.00 \\
(63.43) \mathrm{ab}\end{array}$ \\
\hline 16 & Ferrous oxide $\left(\mathrm{Fe}_{2} \mathrm{O}_{3}\right) 10 \mathrm{ppm}$ & $\begin{array}{c}63.33 \\
(52.73) \mathrm{ab}\end{array}$ & $\begin{array}{c}23.33 \\
(28.88) \mathrm{bcd}\end{array}$ & $\begin{array}{c}0.00 \\
(0.00)\end{array}$ & $\begin{array}{c}0.00 \\
(0.00)\end{array}$ & $\begin{array}{c}86.67 \\
(68.58) a\end{array}$ \\
\hline 17 & Ferrous oxide $\left(\mathrm{Fe}_{2} \mathrm{O}_{3}\right) 20 \mathrm{ppm}$ & $\begin{array}{c}36.67 \\
\text { (37.27)cdef }\end{array}$ & $\begin{array}{c}33.33 \\
(35.26) \mathrm{abc}\end{array}$ & $\begin{array}{c}10.00 \\
(18.43)\end{array}$ & $\begin{array}{c}0.00 \\
(0.00)\end{array}$ & $\begin{array}{c}80.00 \\
(68.43) \mathrm{ab}\end{array}$ \\
\hline 18 & Ferrous oxide $\left(\mathrm{Fe}_{2} \mathrm{O}_{3}\right) 50 \mathrm{ppm}$ & $\begin{array}{c}33.33 \\
\text { (35.26)def }\end{array}$ & $\begin{array}{c}33.33 \\
(35.26) \mathrm{abc}\end{array}$ & $\begin{array}{c}6.67 \\
(14.96)\end{array}$ & $\begin{array}{c}0.00 \\
(0.00)\end{array}$ & $\begin{array}{c}73.33 \\
(58.91) a b\end{array}$ \\
\hline 19 & Ferroous oxide $\left(\mathrm{Fe}_{2} \mathrm{O}_{3}\right) 100$ ppm & $\begin{array}{c}40.00 \\
(39.23) \mathrm{cde}\end{array}$ & $\begin{array}{c}16.67 \\
(24.09) \mathrm{cde}\end{array}$ & $\begin{array}{c}10.00 \\
(18.43)\end{array}$ & $\begin{array}{c}0.00 \\
(0.00)\end{array}$ & $\begin{array}{c}73.33 \\
(58.91) \mathrm{ab}\end{array}$ \\
\hline 20 & Ferrous oxide $\left(\mathrm{Fe}_{2} \mathrm{O}_{3}\right) 500 \mathrm{ppm}$ & $\begin{array}{c}30.00 \\
\text { (33.21)def }\end{array}$ & $\begin{array}{c}23.33 \\
\text { (28.88)bcde }\end{array}$ & $\begin{array}{c}13.33 \\
(21.42)\end{array}$ & $\begin{array}{c}0.00 \\
(0.00)\end{array}$ & $\begin{array}{c}73.33 \\
(58.91) a b\end{array}$ \\
\hline
\end{tabular}




\begin{tabular}{|c|l|c|c|c|c|c|}
\hline $\mathbf{2 1}$ & $B b$ without nano & $\begin{array}{c}23.33 \\
(28.88) \text { efg }\end{array}$ & $\begin{array}{c}16.67 \\
(24.09) \mathrm{cde}\end{array}$ & $\begin{array}{c}13.33 \\
(21.42)\end{array}$ & $\begin{array}{c}0.00 \\
(0.00)\end{array}$ & $\begin{array}{c}60.00 \\
(50.77) \mathrm{b}\end{array}$ \\
\hline $\mathbf{2 2}$ & Control & 6.67 & 6.67 & 3.33 & 0.00 & 16.67 \\
& & $(14.96) \mathrm{g}$ & $(1496) \mathrm{e}$ & $(10.52)$ & $(0.00)$ & $(24.09) \mathrm{c}$ \\
\hline & C.D. & 6.427 & 8.859 & 6.427 & & 10.754 \\
\hline & SE(m) & 2.247 & 3.098 & 2.247 & & 3.761 \\
\hline & SE(d) & 3.178 & 4.381 & 3.178 & & 5.318 \\
\hline & C.V. & 12.234 & 25.848 & 88.588 & & 10.856 \\
\hline
\end{tabular}

Figures in parentheses are arcsine transformed values Alphabets indicating Duncan Multiple Range Test (DMRT)

Table.2 Influence of different nanoparticles on the efficacy of B.bassiana against S.litura at different concentrations during the year 2017

\begin{tabular}{|c|c|c|c|c|c|c|}
\hline \multirow[t]{2}{*}{ S.No. } & \multirow[t]{2}{*}{ Name of the Treatment } & \multicolumn{5}{|c|}{ S.litura Per cent mortality } \\
\hline & & $120 \mathrm{~h}$ & $144 h$ & $168 \mathrm{~h}$ & $192 \mathrm{~h}$ & Cumulative \\
\hline 1 & $\begin{array}{l}\text { Magnesium oxide }(\mathrm{MgO}) 10 \\
\text { ppm }\end{array}$ & $\begin{array}{c}43.33 \\
(41.17) \mathrm{bcd}\end{array}$ & $\begin{array}{c}23.33 \\
\text { (28.88)bcdef }\end{array}$ & $\begin{array}{c}6.67 \\
(14.96) \mathrm{abc}\end{array}$ & $\begin{array}{c}0.00 \\
(0.00)\end{array}$ & $\begin{array}{c}73.33 \\
(58.91) \text { bcde }\end{array}$ \\
\hline 2 & $\begin{array}{l}\text { Magnesium oxide }(\mathrm{MgO}) 20 \\
\text { ppm }\end{array}$ & $\begin{array}{c}43.33 \\
(41.17) \mathrm{bcd}\end{array}$ & $\begin{array}{c}30.00 \\
(33.21) \text { abcde }\end{array}$ & $\begin{array}{c}6.67 \\
(14.96) \mathrm{abc}\end{array}$ & $\begin{array}{c}0.00 \\
(0.00)\end{array}$ & $\begin{array}{c}80.00 \\
(63.43) \mathrm{abcd}\end{array}$ \\
\hline 3 & $\begin{array}{l}\text { Magnesium oxide }(\mathrm{MgO}) 50 \\
\text { ppm }\end{array}$ & $\begin{array}{c}80.00 \\
(63.43) \mathrm{a}\end{array}$ & $\begin{array}{c}13.33 \\
(21.42) \mathrm{ef}\end{array}$ & $\begin{array}{c}0.00 \\
(0.00) \mathrm{c}\end{array}$ & $\begin{array}{c}0.00 \\
(0.00)\end{array}$ & $\begin{array}{c}93.33 \\
(75.04) \mathrm{a}\end{array}$ \\
\hline 4 & $\begin{array}{l}\text { Magnesium oxide }(\mathrm{MgO}) 100 \\
\text { ppm }\end{array}$ & $\begin{array}{c}46.67 \\
(43.09) \mathrm{bc}\end{array}$ & $\begin{array}{c}30.00 \\
(33.21) \text { abcde }\end{array}$ & $\begin{array}{c}10.00 \\
(18.43) \mathrm{abc}\end{array}$ & $\begin{array}{c}0.00 \\
(0.00)\end{array}$ & $\begin{array}{c}86.67 \\
(68.58) \mathrm{ab}\end{array}$ \\
\hline 5 & $\begin{array}{l}\text { Magnesium oxide }(\mathrm{MgO}) 500 \\
\text { ppm }\end{array}$ & $\begin{array}{c}33.33 \\
(35.26) \text { cdef }\end{array}$ & $\begin{array}{c}30.00 \\
\text { (33.21)abcde }\end{array}$ & $\begin{array}{c}13.33 \\
(21.42) \mathrm{abc}\end{array}$ & $\begin{array}{c}0.00 \\
(0.00)\end{array}$ & $\begin{array}{c}76.67 \\
(61.12) \mathrm{abcd}\end{array}$ \\
\hline 6 & Calcium oxide $(\mathrm{CaO}) 10$ ppm & $\begin{array}{l}26.67 \\
\text { (31.09)def }\end{array}$ & $\begin{array}{c}43.33 \\
(41.17) \mathrm{a}\end{array}$ & $\begin{array}{c}10.00 \\
(18.43) \mathrm{abc}\end{array}$ & $\begin{array}{c}0.00 \\
(0.00)\end{array}$ & $\begin{array}{r}80 \\
(63.4\end{array}$ \\
\hline 7 & Calci & $\begin{array}{c}76.67 \\
(61.12) \mathrm{a}\end{array}$ & $\begin{array}{c}16.67 \\
(21.09) \mathrm{cdef}\end{array}$ & $\begin{array}{c}0.00 \\
(0.00) \mathrm{c}\end{array}$ & $\begin{array}{c}0.00 \\
(0.00)\end{array}$ & $\begin{array}{r}93 \\
(75\end{array}$ \\
\hline 8 & Calcil & $\begin{array}{c}33.33 \\
(35.26) \text { cdef }\end{array}$ & $\begin{array}{c}40.00 \\
(39.23) \mathrm{ab}\end{array}$ & $\begin{array}{c}10.00 \\
(18.43) \mathrm{abc}\end{array}$ & $\begin{array}{c}0.00 \\
(0.00)\end{array}$ & $\begin{array}{r}83 \\
(65.9\end{array}$ \\
\hline 9 & e $(\mathrm{CaO}) 100 \mathrm{ppm}$ & $\begin{array}{c}30.00 \\
(33.21) \text { cdef }\end{array}$ & $\begin{array}{c}30.00 \\
\text { (33.21)abcde }\end{array}$ & $\begin{array}{c}16.67 \\
(24.09) \mathrm{ab}\end{array}$ & $\begin{array}{c}0.00 \\
(0.00)\end{array}$ & $\begin{array}{c}76.67 \\
(61.12) \mathrm{abcd}\end{array}$ \\
\hline 10 & $\mathrm{de}(\mathrm{CaO}) 500 \mathrm{ppm}$ & $\begin{array}{c}33.33 \\
(35.26) \text { cdef }\end{array}$ & $\begin{array}{c}30.00 \\
\text { (33.21)abcde }\end{array}$ & $\begin{array}{c}0.00 \\
(0.00) \mathrm{c}\end{array}$ & $\begin{array}{c}0.00 \\
(0.00)\end{array}$ & $\begin{array}{c}63.33 \\
\text { (52.73)def }\end{array}$ \\
\hline 11 & $(\mathrm{ZnO})$ & $\begin{array}{c}36.67 \\
(37.27) \text { bcde }\end{array}$ & $\begin{array}{c}20.00 \\
\text { (26.57)cdef }\end{array}$ & $\begin{array}{c}10.00 \\
(18.43) \mathrm{abc}\end{array}$ & $\begin{array}{c}0.00 \\
(0.00)\end{array}$ & $\begin{array}{c}83.33 \\
(65.91) \mathrm{abc}\end{array}$ \\
\hline 12 & e $(\mathrm{ZnO})$ & $\begin{array}{c}36.67 \\
(37.27) \text { bcde }\end{array}$ & $\begin{array}{c}36.67 \\
(37.27) \mathrm{abc}\end{array}$ & $\begin{array}{c}3.33 \\
(10.52) \mathrm{bc}\end{array}$ & $\begin{array}{c}0.00 \\
(0.00)\end{array}$ & $\begin{array}{c}76.67 \\
\text { (61.12)abcd }\end{array}$ \\
\hline 13 & $(\mathrm{ZnO})$ & $\begin{array}{c}40.00 \\
(39.23) \text { bcde }\end{array}$ & $\begin{array}{c}26.67 \\
(31.09) \text { abcde }\end{array}$ & $\begin{array}{c}10.00 \\
(18.43) \mathrm{abc}\end{array}$ & $\begin{array}{c}0.00 \\
(0.00)\end{array}$ & $\begin{array}{c}76.67 \\
\text { (61.12)abcd }\end{array}$ \\
\hline 14 & O) $100 \mathrm{ppm}$ & $\begin{array}{c}30.00 \\
(33.21) \text { cdef }\end{array}$ & $\begin{array}{c}26.67 \\
\text { (31.09)abcde }\end{array}$ & $\begin{array}{c}20.00 \\
(26.57) \mathrm{a}\end{array}$ & $\begin{array}{c}0.00 \\
(0.00)\end{array}$ & $\begin{array}{c}76.67 \\
(61.12) \mathrm{abcd}\end{array}$ \\
\hline 15 & $\mathrm{Zir}$ & $\begin{array}{c}30.00 \\
(33.21) \text { cdef }\end{array}$ & $\begin{array}{c}23.33 \\
\text { (28.88)bcdef }\end{array}$ & $\begin{array}{c}10.00 \\
(18.43) \mathrm{abc}\end{array}$ & $\begin{array}{c}0.00 \\
(0.00)\end{array}$ & $\begin{array}{c}63.33 \\
\text { (52.73)def }\end{array}$ \\
\hline 16 & Ferrous oxide $\left(\mathrm{Fe}_{2} \mathrm{O}_{3}\right) 10 \mathrm{ppm}$ & $\begin{array}{c}53.33 \\
(46.91) \mathrm{bd}\end{array}$ & $\begin{array}{c}33.33 \\
(35.26) \mathrm{abcd}\end{array}$ & $\begin{array}{c}10.00 \\
(18.43) \mathrm{abc}\end{array}$ & $\begin{array}{c}0.00 \\
(0.00)\end{array}$ & $\begin{array}{c}80.00 \\
(63.43) \mathrm{abcd}\end{array}$ \\
\hline 17 & Ferrous oxide $\left(\mathrm{Fe}_{2} \mathrm{O}_{3}\right) 20 \mathrm{ppm}$ & $\begin{array}{c}36.67 \\
(37.27) \text { bcde }\end{array}$ & $\begin{array}{c}20.00 \\
\text { (26.57)cdef }\end{array}$ & $\begin{array}{c}10.00 \\
(18.43) \mathrm{abc}\end{array}$ & $\begin{array}{c}0.00 \\
(0.00)\end{array}$ & $\begin{array}{c}66.67 \\
\text { (54.74)cdef }\end{array}$ \\
\hline
\end{tabular}




\begin{tabular}{|c|c|c|c|c|c|c|}
\hline 18 & Ferrous oxide $\left(\mathrm{Fe}_{2} \mathrm{O}_{3}\right) 50 \mathrm{ppm}$ & $\begin{array}{c}36.67 \\
37.27) \text { bcde }\end{array}$ & $\begin{array}{c}23.33 \\
(28.88) \text { bcdef }\end{array}$ & $\begin{array}{c}6.67 \\
(14.96) \mathrm{abc}\end{array}$ & $\begin{array}{c}0.00 \\
(0.00)\end{array}$ & $\begin{array}{c}66.67 \\
(54.74) \text { cdef }\end{array}$ \\
\hline 19 & $\begin{array}{l}\text { Ferroous oxide }\left(\mathrm{Fe}_{2} \mathrm{O}_{3}\right) 100 \\
\text { ppm }\end{array}$ & $\begin{array}{c}23.33 \\
(28.88) \text { ef }\end{array}$ & $\begin{array}{c}33.33 \\
(35.26) \text { abcd }\end{array}$ & $\begin{array}{c}0.00 \\
(0.00) \mathrm{c}\end{array}$ & $\begin{array}{c}0.00 \\
(0.00)\end{array}$ & $\begin{array}{c}56.67 \\
(58.83) \text { ef }\end{array}$ \\
\hline 20 & Ferrous oxide $\left(\mathrm{Fe}_{2} \mathrm{O}_{3}\right) 500 \mathrm{ppm}$ & $\begin{array}{c}16.67 \\
(24.09) f g\end{array}$ & $\begin{array}{c}33.33 \\
(35.26) \text { abcd }\end{array}$ & $\begin{array}{c}6.67 \\
(14.96) \mathrm{abc}\end{array}$ & $\begin{array}{c}0.00 \\
(0.00)\end{array}$ & $\begin{array}{c}56.67 \\
(58.83) \text { ef }\end{array}$ \\
\hline 21 & $B b$ without nano & $\begin{array}{l}23.33 \\
(28.88) \text { ef }\end{array}$ & $\begin{array}{c}20.00 \\
(26.57) \mathrm{cdef}\end{array}$ & $\begin{array}{c}10.00 \\
(18.43) \mathrm{abc}\end{array}$ & $\begin{array}{c}0.00 \\
(0.00)\end{array}$ & $\begin{array}{c}53.33 \\
(46.91) f\end{array}$ \\
\hline 22 & Control & $\begin{array}{c}3.33 \\
(10.52) \mathrm{g}\end{array}$ & $\begin{array}{c}6.67 \\
(14.96) \mathrm{f}\end{array}$ & $\begin{array}{c}3.33 \\
(10.52) \mathrm{bc}\end{array}$ & $\begin{array}{c}0.00 \\
(0.00)\end{array}$ & $\begin{array}{c}13.33 \\
(21.42) \mathrm{g}\end{array}$ \\
\hline & C.D. & 9.089 & 8.859 & 7.604 & - & 9.532 \\
\hline & $\mathrm{SE}(\mathrm{m})$ & 3.178 & 3.098 & 2.659 & - & 3.333 \\
\hline & $\mathrm{SE}(\mathrm{d})$ & 4.495 & 4.381 & 3.761 & - & 4.714 \\
\hline & C.V. & 14.89 & 20.007 & 58.456 & - & 8.056 \\
\hline
\end{tabular}

Figures in parentheses are arcsine transformed values Alphabets indicating Duncan Multiple Range Test (DMRT)

The highest S.itura mortality of 76.67 per cent was recorded with $\mathrm{MgO}$ at $50 \mathrm{ppm}$, followed by 73.33 per cent with $\mathrm{CaO}$ at $20 \mathrm{ppm}, 63.33$ per cent with $\mathrm{FeO} 10 \mathrm{ppm}$ and 56.67 per cent with $\mathrm{ZnO} 10 \mathrm{ppm}$. Where as in B.bassiana without nanomaterial based it was recorded as 43.33 per cent and in control it was 6.67 per cent.

The mortality per cent after 144 hours of the treatment ranged from 6.67 to 46.67 per cent in different treatments. The highest per cent mortality 46.67 per cent was recorded with $\mathrm{CaO}$ at $10 \mathrm{ppm}$. The per cent mortality 168 hours after treatment was ranged between 3.33 and 16.67 per cent, and the mortality per cent was gradually decreased.

The cumulative S.itura larval mortality was ranged between 16.67 and 90.00 per cent in different treatments. The highest per cent mortality (90.00) was recorded with $\mathrm{MgO}$ based B.bassiana at $50 \mathrm{ppm}$ which is on par with $\mathrm{CaO} 20 \mathrm{ppm}$ and $\mathrm{FeO} 10 \mathrm{ppm}$ based N.rileyi, followed by $\mathrm{ZnO}$ at $10 \mathrm{ppm}$ (83.33). Where as in control it was 16.67 per cent.

During 2017, the mortality of S.itura after 120 hours of the treatment recorded was ranged from 33.33 to 80.00 with $\mathrm{MgO}$ based B.bassiana, 26.67 to 76.67 with $\mathrm{CaO}$ based
B.bassiana, 16.67 and 53.00 with $\mathrm{FeO}$ based B.bassiana and 30.00 and 40.00 per cent with $\mathrm{ZnO}$ based B.bassiana. The highest mortality per cent 80.00 was recorded with $\mathrm{MgO}$ at 50 ppm, followed by 76.67 per cent with $\mathrm{CaO}$ at 20 ppm, 53.33 per cent with $\mathrm{FeO} 10 \mathrm{ppm}$ and 40.00 per cent with $\mathrm{ZnO} 50 \mathrm{ppm}$. Where as in B.bassiana without nanomaterial based it was recorded as 23.33 per cent and in control it was 3.33 per cent.

The mortality S.itura after 144 hours of the treatment ranged from 6.67 to 43.33 per cent in different treatments. The highest S.itura mortality of 43.33 per cent was recorded with $\mathrm{CaO}$ at $10 \mathrm{ppm}$. The S.itura mortality 168 hours after treatment was ranged between 0 and 20.00 per cent and later the mortality per cent was gradually decreased.

The cumulative S.itura mortality was ranged from 13.33 per cent to 93.33 per cent in different treatments. The highest S.itura mortality (93.33) was recorded with $\mathrm{CaO}$ based B.bassiana at $20 \mathrm{ppm}$ and $\mathrm{MgO} 50$ ppm, followed by (80.00) FeO $10 \mathrm{ppm}$ based B.bassiana and followed by $\mathrm{ZnO} 10 \mathrm{ppm}$ (83.33) based B.bassiana. Where as in B.bassiana without nanoparticles was 53.33 per cent and in control it was 13.33 per cent. 
The results revealed that, highest S.itura mortality was recorded with $\mathrm{MgO} @ 50$ ppm, $\mathrm{CaO} 20 \mathrm{ppm}$ fortified growth media for $B t$ similarly $\mathrm{MgO} @ 50$ ppm, $\mathrm{CaO} 20$ ppm was effective for enhancing the activity of B.bassiana against S.litura. The B.bassiana grown in nanoparticles enriched media were tested against $3^{\text {rd }}$ instar larvae of S.litura under laboratory conditions.

The results revealed that the significant highest per cent mortality was observed at $120 \mathrm{~h}$ with B.bassiana grown under nanoparticles fortified $\mathrm{CaO}$ at $20 \mathrm{ppm}, \mathrm{MgO}$ at $50 \mathrm{ppm}, \mathrm{FeO}$ at $10 \mathrm{ppm}$ and $\mathrm{ZnO}$ at $20 \mathrm{ppm}$ enriched biopesticide when compared with biopesticid without nanoparticles as well as control. The studies of Valicente et al., (2010) on the influence of mineral salts of $\mathrm{FeSO}_{4}$, $\mathrm{ZnSO}_{4}, \mathrm{MnSO}_{4}$ and $\mathrm{MgSO}_{4}$ when added to LBA media at a concentration of $0.002 \mathrm{~g}$, $0.02 \mathrm{~g}, 0.02 \mathrm{~g}$ and $0.03 \mathrm{~g}$ respectively and these resulted in an increased number of viable spores $2 \times 10^{8}$ cells $/ \mathrm{ml}$ of $B t$ compared to control and as well as reported higher efficacy of 60 per cent mortality against first instar larvae of S.frugiperda under laboratory conditions. Similarly Namasivayam et al., (2013) observed the nano sized copper coated chitosan showed the distinct effect on the growth of N.rileyi under field conditions.

\section{Summary}

The B.bassiana grown under nano enriched media was tested against $3^{\text {rd }}$ instar larvae of S.litura under laboratory conditions. The results revealed that the significant highest per cent mortality of S.litura was observed at $120 \mathrm{~h}$ after treatment with B.bassiana grown under $\mathrm{CaO}$ at $20 \mathrm{ppm}, \mathrm{MgO}$ at $50 \mathrm{ppm}, \mathrm{FeO}$ at $10 \mathrm{ppm}$ and $\mathrm{ZnO}$ at $20 \mathrm{ppm}$ nanomaterial enriched media when compared with biopesticides without nanoparticles as well as control.

\section{References}

Ahmad, M., Saleem, M. A and Ahmad, M. 2005. Time oriented mortality in leaf army worm, Spodoptera litura (Fab.) (Lepidoptera: Noctuidae) by some new chemistry insecticides. Pakistan Entomology. 27 (1): 67-70.

Armes, N. J., Wightman, J.A., Jadhav, D.R. and Ranga Rao, G.V. 1997. Status of insecticide resistance in Spodoptera litura in Andhra Pradesh, India. Pesticide Science, 50: 240-248.

Basu, A. C. 1981. Effect of different foods on the larval and post larval development of moth of Prodenia litura (Fab.). Journal of Bombay Natural History Society; 44: 275-288.

Hafez, M., Zaki, F.N., Moursy, A and Sabbour, M. 1994. Biological effects of the entomopathogenic fungus, Beauveria bassiana on the potato tuber moth, Phthormaea operculella (Seller). Journal of Islamic Academy Sciences. 57: 167-168.

Kumar, K and Chapman, R. B. 2006. Sublethal effects of insecticides on the diamondback moth Plutella xylostella (L.). Pesticide Science. 15: 344-352.

Mousa, M. A., Zaker, M. A and Koppy, F. 1980. Abundance of cotton leaf worm, Prodenia litura (Fab.) in relation to host plants Host plants and their effect on biology. Bulletin of Entomological Society, Egypte. 44: 241-251.

Muthukrishnan, N., Ganapathy, N., Nalini, R and Rajendran, R. 2005. Pest Management in Horticultural Crops. New Madura Publishers, Madurai. PP. 325.

Namasivayam, K.R., Bharani, R.S.A and Ansari M.R. 2013. Natural Occurrence of Potential Fungal Biopesticide Nomuraea rileyi (Farlow) Samson Associated with Agriculture Fields of Tamil Nadu, India and it's Compatibility with Metallic 
Nanoparticles. Journal of Biofertilizers \& Biopesticides. 4: 132.

Sahayaraj, K., Selvarj, P. and Balasubramanian, R. 2007. Cell mediated immune response of Helicoverpa armigera Hubner and Spodoptera litura Fabricius to Fern Phytoecdysteron. Journal of Entomology, 4: 289-298.

Sasidharan, K. R and Varma, R.V. 2005. Laboratory evaluation of Beauveria bassiana (Balsamo) Vuillemin against Indarbela quadrinotata Walker (Lepidoptera: Metarbelidae) - a key pest of Casuarina equisetifolia L. in Tamil Nadu. Journal of Biological Control. 19: 197- 200.

Stark, J. D. and Banks, J. E. 2003. Population- level effects of pesticides and other toxicants on arthropods. Annual Review of Entomology, 48: 505-19.

Suchea, M., Christoulakis, S., Moschovis, K., Katsarakis, N and Kiriakidis, G. 2006. $\mathrm{ZnO}$ transparent thin films for gas sensor applications. Thin Solid Films. 515: 551-554.

Valicente, F.H., Tuelher, E.D.S, Liete, M.I.S, Freire, F.L and Vieira, C.M. 2010.Production of Bacillus thuringiensis biopesticide using commercial lab medium and agricultural by-products as nutrient sources. Revista Brasileira de Milho e Sorgo. 9 (1): 1-11.

\section{How to cite this article:}

Gayathri, M., N.C. Venkateswarlu, T. Murali Krishna and Devaki, K. 2018. Evaluation of the Beauveria bassiana Grown under Nanomaterial Enriched Media for its Relative Efficacy against S. litura under Laboratory Conditions. Int.J.Curr.Microbiol.App.Sci. 7(12): 2017-2024. doi: https://doi.org/10.20546/ijcmas.2018.712.232 